\title{
Glass patterns: some contrast effects re-evaluated
}

\author{
Steven C Dakin \\ McGill Vision Research Unit, Department of Ophthalmology, 687 Pine Avenue West, H4-14, Montreal, \\ Quebec H3A 1A1, Canada; e-mail: scdakin@astra.vision.mcgill.ca \\ Received 18 October 1996, in revised form 16 December 1996
}

\begin{abstract}
The relative contrast of features is known to be important in determining if they can be grouped. Two manipulations of feature contrast have previously been used to criticise models of visual grouping based on spatial filtering: high-pass filtering and reversal of contrast polarity. The effects of these manipulations are considered in the context of the perception of Glass patterns. It is shown that high-pass filtering elements, whilst destroying structure in the output of low-pass filters, do not significantly disrupt the output of locally band-pass filters. The finding that subjects can perceive structure in Glass patterns composed of high-pass features therefore offers no evidence against such spatial filtering mechanisms. Band-pass filtering models are shown to explain the rotation of perceived structure in Glass patterns composed of opposite contrast features. However, structure is correctly perceived in patterns composed of two 'interleaved' opposite contrast patterns, which is problematic for oriented filtering mechanisms. Two possible explanations are considered: nonlinear contrast transduction prior to filtering, and integration of local orientation estimates from first-order and second-order mechanisms.
\end{abstract}

\section{Introduction}

The spatial filters at the core of most models of early visual processing are linear; they transform the image intensity at a point into a weighted linear combination of neighbouring values. The view that the visual system decomposes the complex twodimensional signal from an image into a linear sum of terms in the frequency domain is convenient for two reasons. First, the assumption of linearity allows the study of the visual system psychophysically through the presentation of patterns that contain only a small number of those frequency terms (eg Campbell and Robson 1968). Second, it allows for computationally efficient simulations of visual processing by Fourier techniquesefficient methods for moving between spatial and frequency based representations.

It has been proposed that spatial filters provide a natural mechanism for visual grouping (eg Watt 1988). If two features fall into the excitatory field of a filter, then its response will be significantly increased or decreased (depending on the polarity of the features). A cluster of such responses, extending between the positions of the two features, signals a group. However, if the average luminance of the two components is close to the mean luminance, they will not produce an elevated response. Since it is the response of these filters that is proposed as a mechanism for grouping spatially distinct features, this suggests a weakness of the mechanism. It should be possible to construct stimuli for which grouping will be eradicated when the contrast of the components is altered in such a way as to destroy the structured output of filters. If the breakdown of grouping predicted by a purely linear system does not correspond to human performance, then a reassessment of the model would be required.

In this paper the problem of grouping is considered in the context of one class of stimuli: Glass patterns (Glass 1969). These textures, an example of which can be seen in figures la and $1 \mathrm{~b}$, are generated by taking one spatially randomly distributed set of dots (or any other small feature), and superimposing a geometrically transformed copy of the set. These textures are perceived as having compelling orientation structure and of being composed of dot pairs (or dipoles) locally aligned in the direction of the 


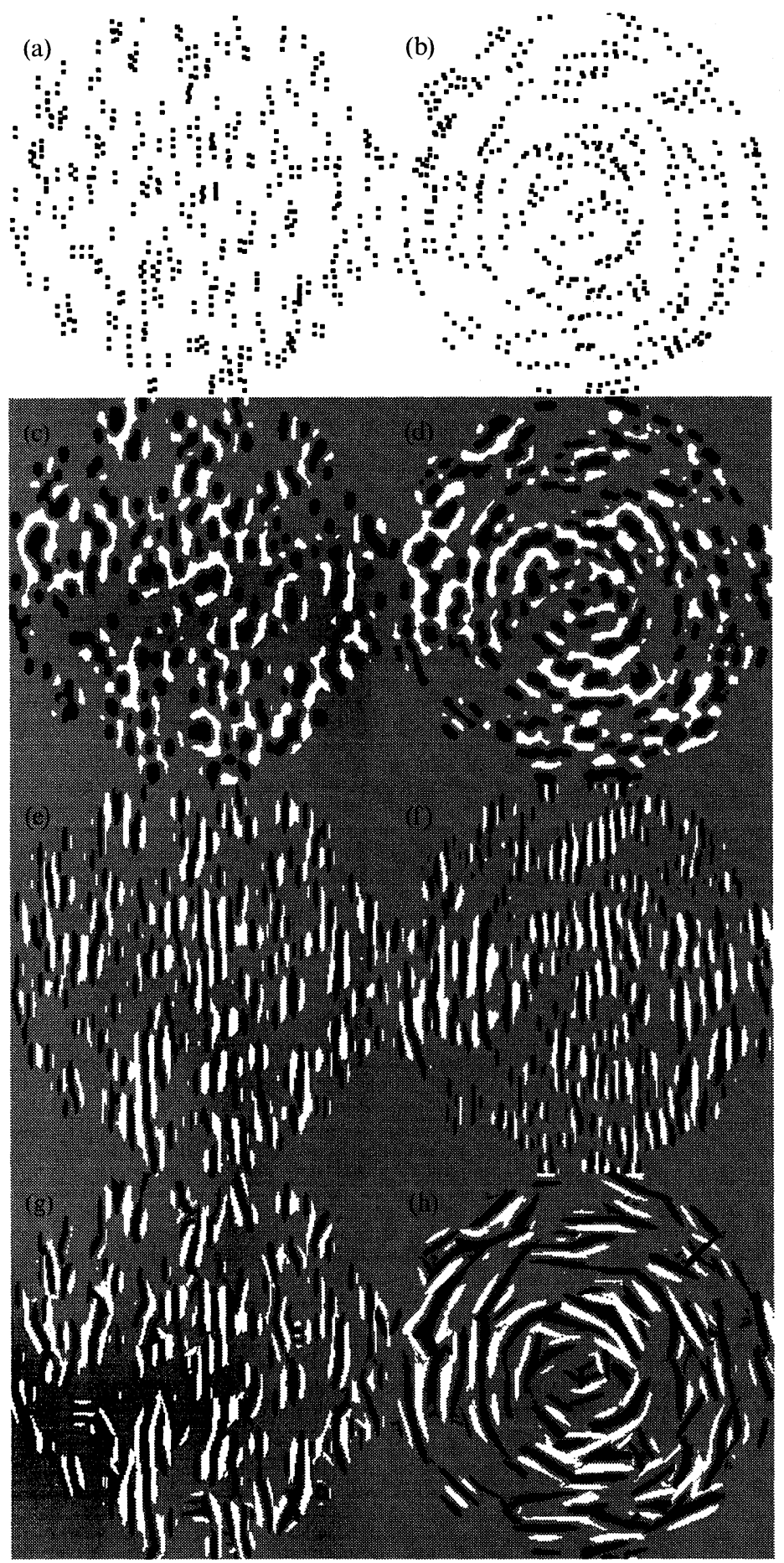

Figure 1. Examples of Glass patterns with (a) translational and (b) rotational structure. (c) - (h) show filtered and thresholded versions of (a), (b). For the purpose of highlighting the resultant structure, grey levels above or below threshold have been replaced with white or black pixels, respectively. The filtering used to generate these patterns was: (c), (d) isotropic, Laplacian-of-Gaussian (LoG); (e), (f) oriented, Difference-of-Gaussian (DoG); (g), (h) 'adaptive', ie point-by-point selection of the most active oriented DoG filter. 
geometric transformation used to generate them. In order to perceive structure in these patterns one must be solving a form of correspondence problem, grouping individual dots into dipoles.

A simple way of performing this grouping operation is through the application of spatial filters (Kass and Witkin 1985; Zucker 1982). Figures 1c and 1d show convolutions of Glass patterns with isotropic Laplacian-of-Gaussian (LoG) filters (followed by thresholding at one grey-level standard deviation above and below the mean). Note that the dark 'blobs' often correspond to grouped collections of dipoles; their orientation reflects the local orientation of the pattern. Another possible filtering mechanism is oriented, Difference-of-Gaussians (DoG) filtering (figures le and 1f). Two-dimensional DoG filters are composed of a band-pass Difference-of-Gaussians in one direction, and a low-pass Gaussian in the orthogonal direction.

Notice that features resulting from DoG filters in figure le are much more elongated because they are sensitive to a narrower range of orientations. It would be desirable to retain this orientational sensitivity, but to be able to derive complex orientation structure, such as rotational fields (figure $1 \mathrm{~b}$ ) which a single DoG filter cannot do (figure 1f). 'Adaptive' oriented filtering (figures 1g and 1h; Dakin, in press) is a simple scheme for combining the outputs of filters at multiple orientations. The filter output at each orientation is squared and a small degree of Gaussian blurring applied, to form an estimate of that filter's 'local power'. Finally, a single new image is constructed by selecting, on a pixel-by-pixel basis, the original filter output whose local power is greatest at that position. The blobs that result from running a rotational Glass pattern through the adaptive filtering scheme (figure $1 \mathrm{~h}$ ) are elongated but accurately reflect local orientation structure.

In order to quantitatively assess the performance of such filtering models one must address two key problems: how to produce estimates of dipole orientation from filtered images, and how to select an appropriate filter size (Prazdny 1986). In order to measure dipole orientation, one must first delineate discrete features from the filtered image. Thresholding the image (eg figure lc) produces 'blobs' whose orientations may be measured by means of an image description scheme (Watt 1991). This produces a sentence-based representation reminiscent of the 'primal sketch' (Marr 1976) in which feature orientation is explicit. With respect to the first problem, that of scale selection, it is known that orientation information is of primary importance in the perception of Glass patterns. Variation in dipole orientation, but not length, determines their discriminability from noise (Caelli and Julesz 1979). If one assumes that the goal of deriving structure from oriented texture, such as Glass patterns, is to accurately assess local orientation then one way to maximise confidence in these estimates is to select the spatial scale which minimises the variability of orientation data (Dakin, in press). Orientation variability may be assessed automatically by deriving an image description, in the manner described above, and estimating the variance of blob orientations. This criterion for scale selection is used throughout this paper to select filter size automatically.

Two types of such contrast phenomena have been widely used to criticise models of visual grouping based on spatial filtering. The first phenomenon is the perceived grouping of spatially high-pass, or 'balanced' dots which (supposedly) do not contain the low-spatial-frequency information that filtering models are sensitive to. Stevens and Brookes (1978) showed that structure is correctly perceived in Glass patterns composed of balanced energy dots (each feature is made up of a bright centre surrounded by dark ring). They claim that whilst we can perceive structure in balanced energy patterns, models based on spatial summation will 'smooth out' individual features and therefore cannot explain the perceived grouping. It will be demonstrated below that band-pass filtering models can correctly predict perceived structure. 
The second phenomenon is, that contrary to the prediction of filtering models, features of opposite contrast polarity lead to grouping under certain circumstances. If the correlated elements comprising a Glass pattern are of opposite contrast polarity then, it is claimed, orientation structure cannot be perceived (Glass and Switkes 1976; Prazdny 1986; Zucker and Davis 1988; Zucker et al 1983). However, Kovacs and Julesz (1992) presented psychophysical data showing that, for dense Glass patterns at least, perceived flow is not destroyed but rotated through $90^{\circ}$. This finding will be shown to be consistent with a model of grouping based on linear spatial filtering.

More complex stimuli have been designed to 'fool' filtering models. Prazdny (1986) pointed out that the correct flow direction is perceived in a texture composed of two 'interleaved' Glass patterns, each with opposite contrast polarity. Elements of these patterns are composed of a sequence of dots with positive, negative, positive, and negative contrast polarities (eg figure 8). Linear filtering of these patterns does not produce the correct structure at any spatial scale, and Prazdny (1986) has taken this as strong evidence against models employing such mechanisms. Later I shall argue that such demonstrations imply the presence of second-order mechanisms in the extraction of orientation from Glass patterns.

\section{Spatially high-pass textures}

Many proposed filtering models for extracting tokens from images use mechanisms that are sensitive only to low spatial frequencies. Figure 2 shows (a) a typical Glass pattern, and (b) its power spectrum, with the boundaries of the sensitivity of a typical horizontal

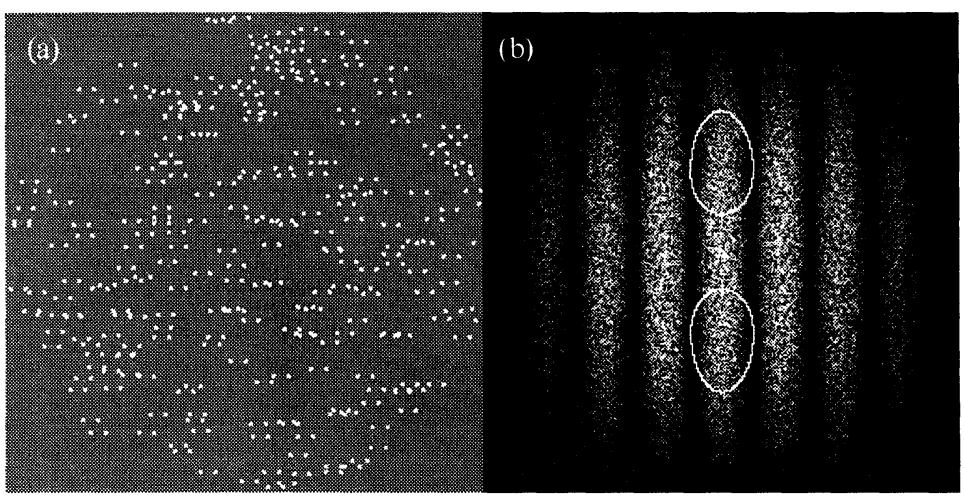

(c)

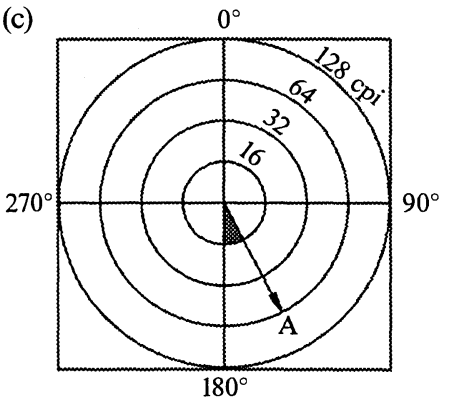

Figure 2. (a) Horizontal Glass pattern with dipole length of 8 pixels. (b) Fourier power spectrum of (a). On this polar plot, distance and orientation from the origin correspond to spatial frequency and orientation, respectively. This is illustrated in (c), which shows point $\mathrm{A}$ at an angle of $150^{\circ}$ and a frequency of 64 cycles per image (cpi). The boundaries superimposed on (b) show the approximate limit of the sensitivity of a DoG filter with peak sensitivity of $41 \mathrm{cpi}$. Note that the boundaries capture high-energy regions of the spectrum, and that horizontal energy (the vertical column centred on the middle of the pattern) is spread over a large range of scales. 
DoG filter superimposed. The latter image is on polar axis: the distance from the centre indicates scale $(0-128$ cycles per image), and the orientation of a point from the centre indicates orientation. Brightness is proportional to the power at that scale/orientation. The vertical stripes of high energy in figure $1 \mathrm{~b}$ are due to the dot spacing of the dipoles. ${ }^{(1)}$ Notice that the power spectrum contains horizontal energy over a wide range of scales, and that that energy extends over the origin. This indicates that low-spatial-frequency information will signal the presence of horizontal structure in the pattern. This could be detected by means of a coarse-scale low-pass filter (eg a Gaussian).

An apparently simple way of testing such models is to remove low-spatial-frequency information from the image by high-pass filtering it. An approximation to the high-pass filtered version of a dot is the 'balanced' dot (Carlson et al 1980). This consists of an inner area with luminance greater than the background, surrounded by a region with luminance less than the background. The inner and outer luminances are selected so that overall average luminance of the feature is equal to the background. Structure may be perceived in patterns composed of these high-pass features, notably for 'Gestalt' grouping (Janez 1984; Palmer 1992) and visual illusions (Carlson et al 1980; but see Garcia-Perez 1991). Such work has been cited as evidence against low-pass filters as mechanisms for visual grouping (eg Beck et al 1987; Palmer 1992; Reed and Wechsler 1990).

Prazdny (1986), however, generated Glass patterns composed of small Laplacian rings and claimed that, even at $1 \mathrm{~s}$ exposure duration, subjects attempting to distinguish between transformation types approached chance levels of performance when background luminance approached the same value as the mean of the Laplacian features. This is entirely contrary to predictions from other grouping studies in which balanced dots have been used and may be interpreted as evidence for a grouping process that involves a degree of spatial summation. It is hard for models based on matching of symbolic tokens (eg Stevens 1978) to accommodate such results, because all features, balanced or otherwise, are identical (as long as they are visible) and should be strongly matched.

Stevens and Brookes (1978) found a result contradictory to Prazdny's (1986) using patterns composed of small balanced dots. Subjects were able to correctly perceive structure in these patterns at exposure durations of as little as $200 \mathrm{~ms}$. Stevens and Brookes claim that the discrepancy between this and Prazdny's result is because features in their patterns were scaled for eccentricity. In order to perceive structure in globally organized Glass patterns it is known that one must be able to see a sufficiently large region of the pattern (Glass 1969) and it is possible that, owing to the effects of retinal eccentricity, the outer portions of Prazdny's fairly large ( $8.25 \mathrm{deg}$ wide) patterns were not visible to subjects. Hence they could not discriminate between globally organized patterns. This interpretation ignores the fact that subjects in Prazdny's study had long enough to use eye movements. Also Stevens and Brookes present no quantitative estimate of the strength of perceived structure in these patterns. These objections aside, the proposition that structure can be perceived in these patterns which are 'devoid of low spatial frequencies' (Stevens and Brookes 1978), will now be considered.

Stevens and Brookes (1978) claim that, since balanced dots provide negligible input to a simple cell whose excitatory receptive field they lie within, the addition of an early nonlinearity in intensity transduction is necessary. Figure 3 a shows a typical balanced pattern and figure $3 \mathrm{~b}$ its convolution with a LoG filter. The LoG produces poorly oriented blobs but does seem to reflect the circular structure. Figure $3 \mathrm{c}$ shows the adaptively filtered image, and figure $3 \mathrm{~d}$ the flow field derived from it. In contradiction to Stevens and Brookes's prediction, band-pass filter outputs do accurately reflect dipole orientation. The adaptive filtering model does nonlinearly combine filter outputs (a peak

(1) If we approximate the bright-dark-bright luminance profile of a dipole with 1.5 cycles of a square-wave grating, then we would expect that, as the scale of analysis of this pattern changed, the energy of this feature would vary periodically. 
selection process is used). However, this is not the reason why it correctly extracts dipole orientation. Figure $4 \mathrm{~b}$ shows that while low spatial frequencies are not present in balanced Glass patterns (indicated by the 'hole' at the origin of the power spectrumcompare to figure 2b) structure persists at higher spatial scales. The intersection of the sensitivity boundary of the DoG filter with regions of high energy indicates that such structure is readily detectable with an appropriately oriented filter. The adaptive mechanism simply selects the DoG filter which derives the correct orientation structure.

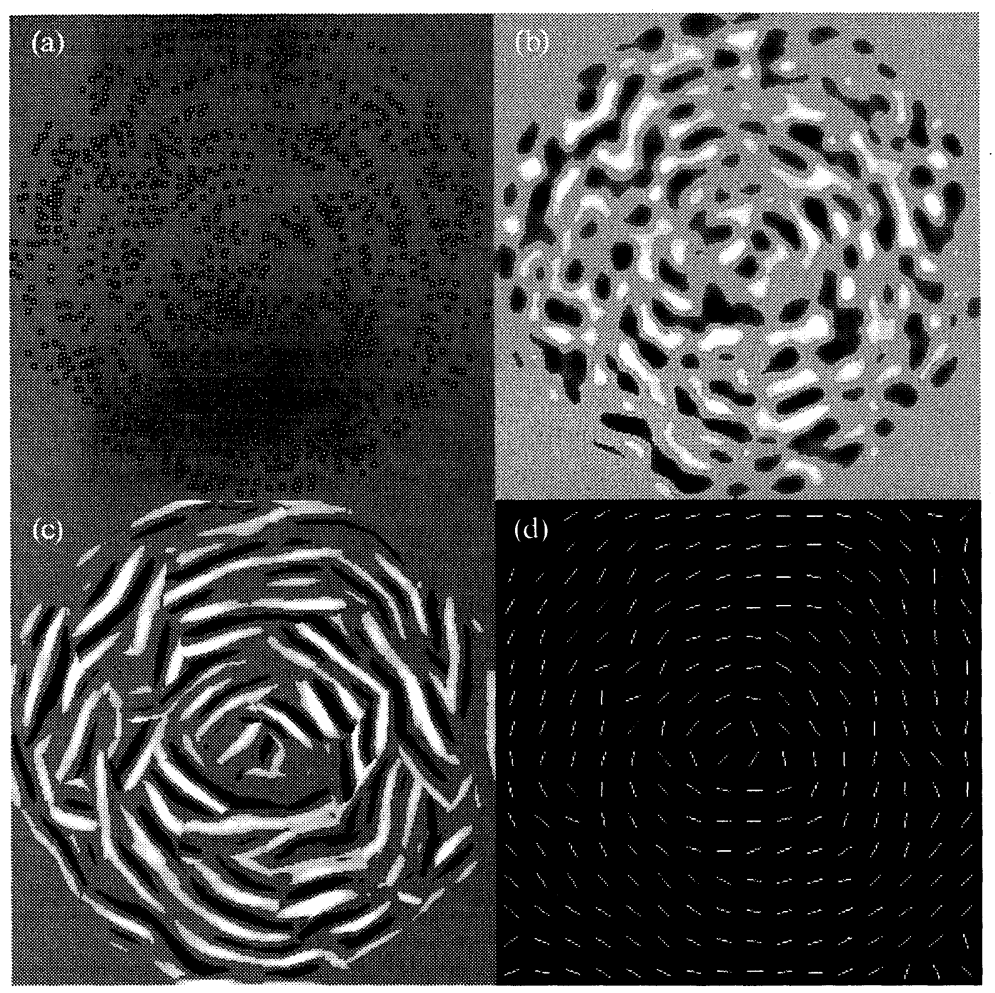

Figure 3. (a) A balanced Glass pattern (contrast enhanced for the purpose of reproduction). (b) LoG filtering poorly enhances orientation structure but output of the adaptive filtering model, (c), does much better. Note highly oriented features and derived flow field (d).

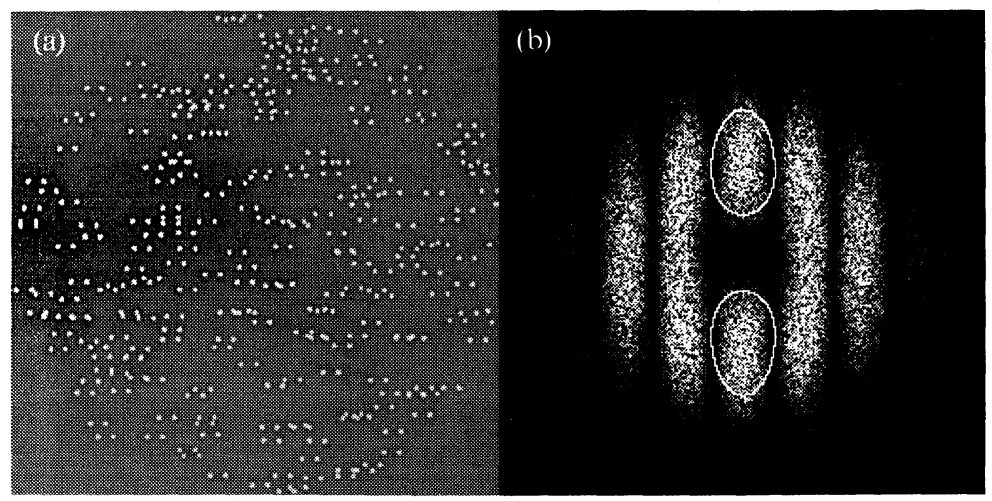

Figure 4. (a) High-pass-filtered horizontal Glass pattern. (b) Fourier power spectrum of (a). Superimposed boundaries show the approximate limit of the sensitivity of a DoG filter with peak sensitivity of 41 cycles per image. Note that the boundaries capture high energy regions of the spectrum. 
To summarise, balanced features specifically disrupt the output of low-pass filtering mechanisms, but models using spatially band-pass filtering have no such problems grouping these features. I conclude that human perception of structure in these patterns offers no evidence against models of visual grouping based on band-pass (oriented or isotropic) filters. Prazdny's (1986) finding that subjects could not see structure in these patterns seems likely to be due to the artifacts pointed out by Stevens and Brookes (1978).

\section{Opposite-contrast Glass patterns}

An opposite-contrast Glass pattern is produced in the same way as an ordinary pattern except that one set of randomly distributed features is of opposite contrast polarity to the second, transformed set. ${ }^{(2)}$ An example is shown in figure 5a. The failure to see horizontal structure in this pattern is explicable in terms of the Fourier power spectrum presented in figure $5 \mathrm{~b}$. There is very little horizontal energy at any spatial scale, indicated by the dark vertical strip centred on the origin.

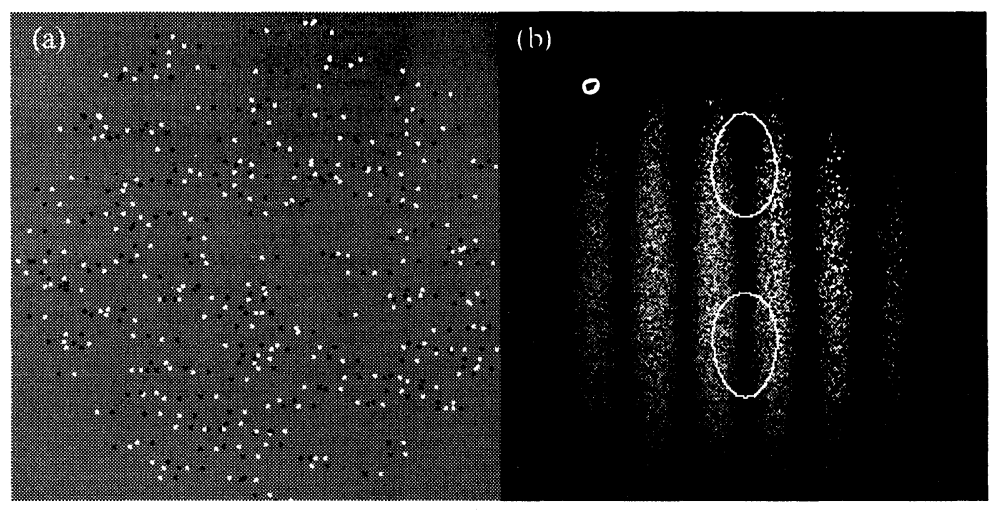

Figure 5. Horizontal Glass pattern, with dipole length of 8 pixels, composed of opposite contrast dots. (b) Fourier power spectrum of (a). Superimposed boundaries show the approximate limit of the sensitivity of a DoG filter with peak sensitivity of 41 cycles per image. Note that the boundaries do not capture high-energy regions of the spectrum.

Prazdny (1986) has claimed that correct structure cannot be seen in opposite-contrast Glass patterns, but the impression gained, from a dilational pattern for example (figure 6b), is usually of a 'petal' or 'spiral' pattern (Glass and Switkes 1976). ${ }^{(3)}$ Glass and Switkes (1976) have stated that this percept is inconsistent with a physiological model based on summation of inputs from Kuffler-type receptive fields. This is because simple cells sum the input from either on-centre or off-centre fields but not from both (Hubel and Wiesel 1967; Tolhurst and Thompson 1975). Thus opposite-contrast dots should not activate simple cells sensitive to the orientation of the dipole and so, according to Glass and Switkes (1976), "the hypothesized physiological mechanism does not appear to explain the observation of spiral-like patterns" (page 71).

These spirals may simply be considered as partial rotations of perceived local orientation. This phenomenon is readily explained by a band-pass spatial filtering mechanism such as the adaptive filtering scheme described. Figures $6 \mathrm{a}$ and $6 \mathrm{~b}$ show two Glass patterns, composed of dot pairs made up of elements of (a) the same and (b) opposite contrast. The operation of an adaptive, oriented filtering mechanism (figures $6 \mathrm{c}$ and $6 \mathrm{~d}$ ) and the derived flow fields (figures $6 \mathrm{e}$ and $6 \mathrm{f}$ ) are also shown. Scale was selected by using the criterion of minimal orientation variance, as described above

(2) In the examples shown the order of the opposite contrast elements is also randomised within each dipole.

(3) Anstis (1970) has shown an analogous phenomenon with random dot cinematograms. 


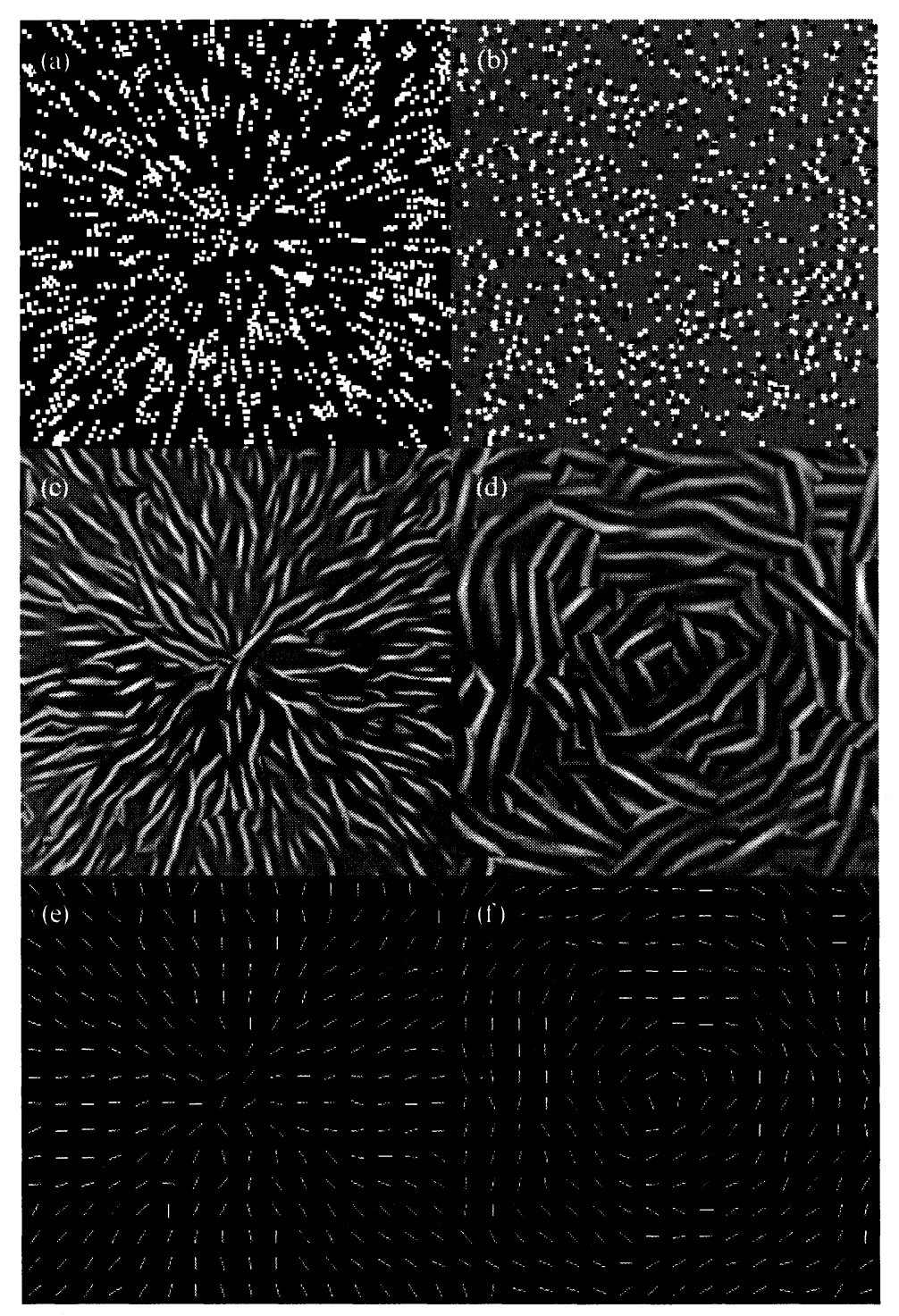

Figure 6. The effect of reversing contrast polarity on the model. (a)-(b) Same-contrast-polarity and opposite-contrast-polarity Glass patterns. (c) - (d) Above patterns filtered at the optimal spatial scale selected by the model. (e) - (f) The derived flow fields. Note that the model predicts a $90^{\circ}$ rotation of local flow in (b).

(Dakin, in press). Note that the spatial scale of figure $6 \mathrm{~d}$ is coarser than for figure $6 \mathrm{c}$. This scale seems to depend on the density of the reverse polarity pattern: the denser the pattern, the finer the scale selected and the stronger the impression of $90^{\circ}$ rotated structure. Figures $6 \mathrm{e}$ and $6 \mathrm{f}$ show the flow fields derived from figures $6 \mathrm{c}$ and $6 \mathrm{~d}$, respectively. The local orientation of figure $6 \mathrm{f}$ is clearly locally orthogonal to figure $6 \mathrm{e}$ in all regions of the texture (which is in accord with one's perception of structure in figure $6 \mathrm{~b}$ ). This is not surprising when considering how the presence of a dipole affects the local statistics of a texture. A same-polarity dipole increases the probability that in moving in the direction of the dipole orientation one will encounter an element of the same brightness, and it is this correlation that a DoG filter highlights. In the case of opposite contrast pairs, the probability of encountering elements of similar 
brightness in the direction of dipole orientation has been greatly reduced. Thus the probability of encountering elements of similar brightness in all other directions is increased. It is this correlation which produces an increase in energy at low spatial frequencies, specifically at orientations orthogonal to the dipole orientation.

Kovacs and Julesz (1992) have examined the conditions under which perception of orthogonal local orientation occurs for Glass patterns. They systematically varied the background luminance of horizontal and vertical translational Glass patterns, as a function of the mean luminance of the dots making up each dipole. Given a pair of dots with luminances $L_{\text {pos }}$ and $L_{\text {neg }}$ (where $L_{\text {neg }}<L_{\text {pos }}$ ), there are three critical ranges of the background luminance, $L_{\text {back }}$ :

1. $L_{\text {neg }}>L_{\text {back }}$ or $L_{\text {pos }}<L_{\text {back }}$ (a similar-contrast-polarity pattern);

2. $L_{\text {neg }}=L_{\text {back }}$ or $L_{\text {pos }}=L_{\text {back }}$ (a random pattern, since only one feature set is visible);

3. $L_{\text {neg }}<L_{\text {back }}<L_{\text {pos }}$ (a reversed-contrast-polarity pattern).

Kovacs and Julesz (1992) measured subjects' accuracy of reporting whether Glass patterns were horizontal or vertical. Data from two subjects and two dot luminances figure 7) show that performance approached $100 \%$ correct for background contrasts producing features of the same polarity (range 1). As background luminance approached the luminance of one dot set (ie only one set was visible-range 2), performance fell to the $50 \%$ level, as expected. However, as background luminance approached the mean of the feature luminances (ie opposite-contrast patterns-range 3), performance fell below the $50 \%$ level, indicating a perception of orientation orthogonal to the true structure (henceforth termed orthogonal-flow perception). Kovacs and Julesz (1992) went on to show that, unlike analogously constructed random-dot cinematograms, this phenomenon cannot be overridden by chromatic cues. Patterns constructed of red dipoles produce the same percept of orthogonal flow when dipoles contain one dot brighter, and the other dimmer, than a green background. Kovacs and Julesz (1992) conclude that it is specifically form perception which is damaged by the lack of luminance cues offered by these patterns.

In order to compare these data to the performance of the adaptive filtering model, a Monte Carlo simulation of the Kovacs and Julesz (1992) task was performed.

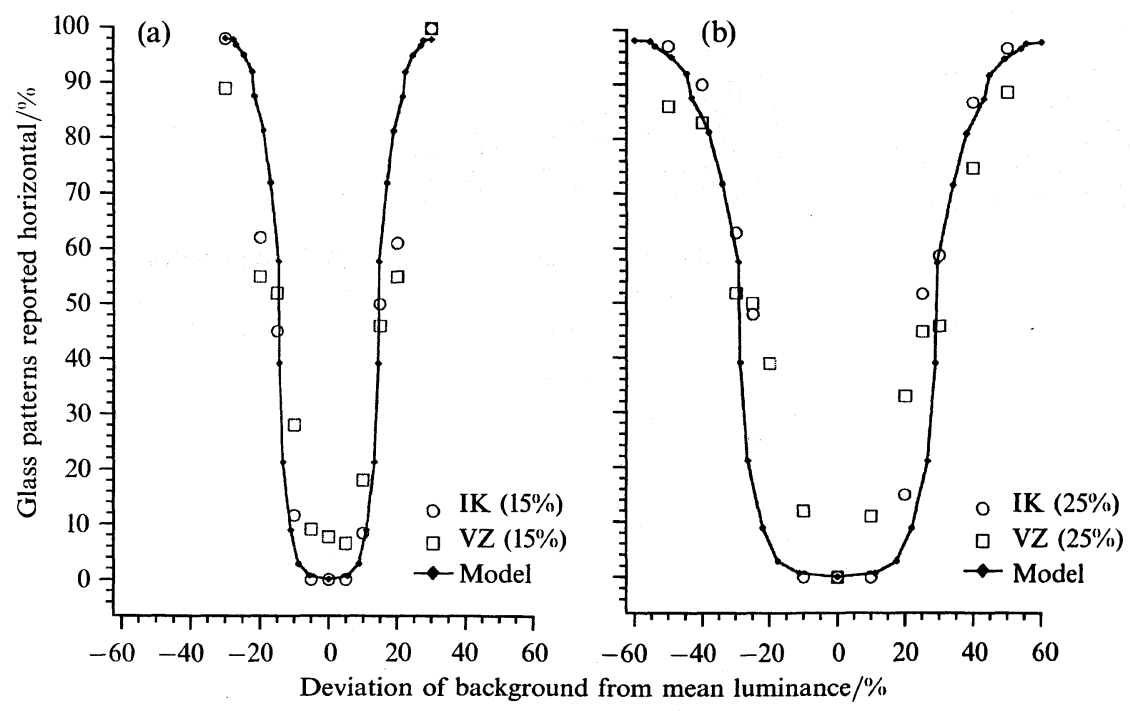

Figure 7. Unfilled symbols show the percentage of Glass patterns classified as horizontal as a function of background luminance, for two subjects, at two levels of mean luminance of correlated features (data are taken from Kovacs and Julesz 1992). The solid lines are predictions from the adaptive filtering model described in the text. 
Glass patterns were generated which were similar to their stimuli; each pattern was a $90 \times 90$ pixels, 256 grey-level image. All patterns were composed of a two-pixel horizontal translation (ie the distance from the centre of an original pixel to the centre of a translated pixel was three pixels). One hundred patterns were generated at 40 levels of background luminance, which ranged from $-2 L_{\text {neg }}$ to $2 L_{\text {pos }}$ in steps of $\left(L_{\text {pos }}-L_{\text {neg }}\right) / 20$. The adaptive filtering model ${ }^{(4)}$ was used to make an estimate of the mean orientation of each pattern, in the range $0^{\circ}-180^{\circ}$. If the reported orientation was between $45^{\circ}$ and $135^{\circ}$, the pattern was classified as vertical, otherwise it was classified as horizontal.

The solid lines in figure 7 show the percentage of patterns classified as horizontal, as a function of the background luminance, compared to human performance (unfilled symbols). Predictions from a single simulation have been matched to data from the two dot luminance conditions by scaling the physical luminances used in the simulations to the appropriate units used in Kovacs and Julesz (1992), but no fitting has been applied to the data. Note that the model shows good agreement with the human subjects at the three contrast ranges of primary interest $\left(\chi^{2}\right.$ measures of the fit are not reported because the standard errors of human data were not available). The model reports $100 \%$ correct for same-polarity patterns, around $50 \%$ when only one feature set is visible, and $0 \%$ correct (ie orthogonal flow; the estimated orientation is consistently $90^{\circ}$ from the true dipole orientation) when pure opposite contrast patterns are presented. The reported perceived rotation of pattern direction seems to be quantitatively explicable in terms of the filtering model described.

\section{Interleaved opposite contrast patterns}

As noted previously, Prazdny (1986) has made two criticisms of explanations of grouping in Glass patterns based on spatial filtering. The first relates to how filter size is automatically selected, which has been considered elsewhere (Dakin, in press). The second is how to deal with a specific type of Glass pattern, composed of two interleaved' patterns of opposite contrast. Figure 8a shows such a pattern where each texture element is composed of four dots with alternating contrast polarity. Subjectively, such textures appear oriented in the direction of dot displacement and do not produce the pattern rotation shown for ordinary opposite-contrast Glass patterns. Prazdny (1986)

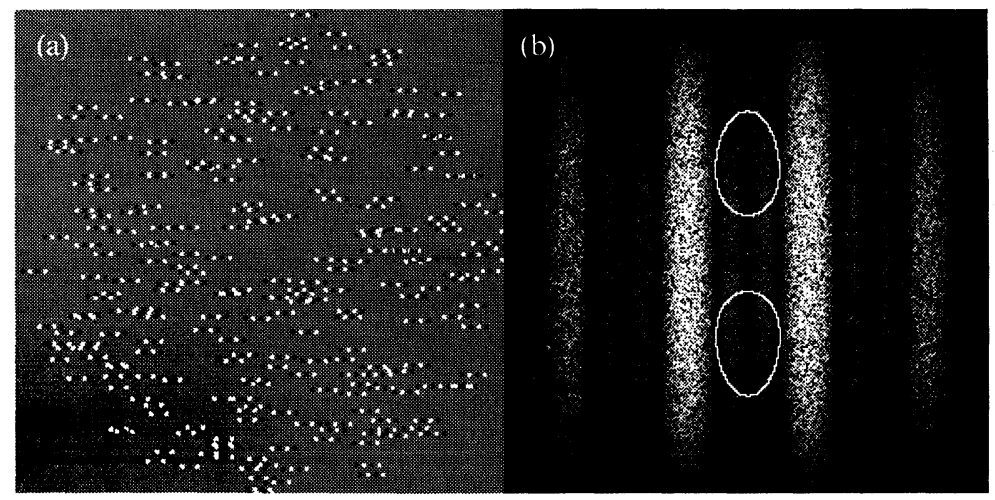

Figure 8. (a) Horizontal Glass pattern, composed of alternating opposite-contrast dots, with a dot separation of two pixels. (b) Fourier power spectrum of (a). Superimposed boundaries show the approximate limit of the sensitivity of a DoG filter with peak sensitivity of 41 cycles per image. Note that the boundaries capture low-energy portions of the spectrum.

(4) The model produced an estimate of mean orientation by thresholding filter outputs at one grey-level standard deviation above and below the mean, constructing a description of each 'blob' by an image description scheme (Watt, 1991), and then averaging blob orientation (allowing for orientational wrap-around). Scale was selected by minimising blob orientation variance. 
states that the only structure visible in band-pass filtered versions of such patterns lies in a direction orthogonal to the perceived direction of flow, which makes explanation of the correctly perceived structure, in terms of linear filtering, problematic. The power spectrum of an interleaved pattern, shown in figure $8 \mathrm{~b}$, confirms that there is very little energy in the horizontal direction across scale, indicated by the faint vertical stripes around the image origin. Figures $9 b$ and $9 c$ confirm that the adaptive filtering model does indeed produce a rotation of flow direction when presented with such a pattern. These patterns can be thought of as balanced patterns that have been specifically formulated to disrupt oriented filtering mechanisms.

The explanation for our veridical perception of structure in these patterns requires a nonlinear transformation of image luminance at some point in processing. Two types of nonlinearity are now considered: an early contrast transduction nonlinearity prior to filtering, and a system combining first-order and second-order estimates of orientation.

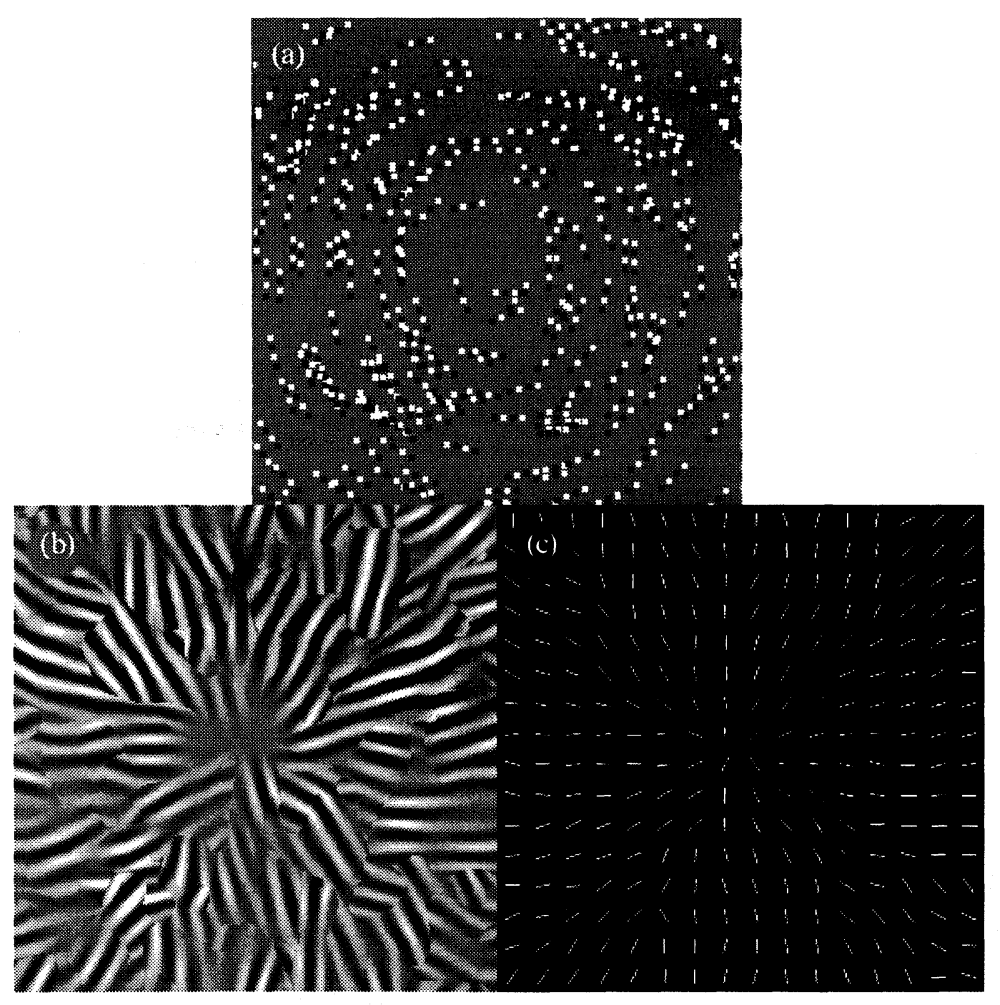

Figure 9. (a) An 'interleaved' opposite-contrast Glass pattern; (b) and (c) the result of running it through the adaptive filtering model. The derived flow field does not correspond to our perception of structure in this pattern.

\subsection{Nonlinear contrast transduction}

There is considerable evidence from psychophysics (eg Legge and Kersten 1983; Morgan et al 1984) and neurophysiology (eg Naka and Rushton 1966; Shapley and Victor 1979) for a compressive nonlinearity modifying luminance prior to filtering. Indeed the utility of an early nonlinearity has already been recognised in a number of models of texture segregation motivated by human psychophysical performance (Graham et al 1992). Different types of nonlinearity have been proposed, such as the sigmoid (Gelb and Wilson 1983), but a particularly computationally simple formulation is the 
H-transform (Morgan et al 1984; Naka and Rushton 1966; Watt 1991), which has the form:

$$
R(x, y)=L(x, y) \frac{R_{\max }}{H+L(x, y)},
$$

where $L(x, y)$ is the image intensity at position $(x, y), R(x, y)$ is the output of the $\mathrm{H}$ transform at that point, $R_{\max }$ is the maximum response, and $H$ is the semisaturation luminance at which $R=0.5 R_{\max }$.

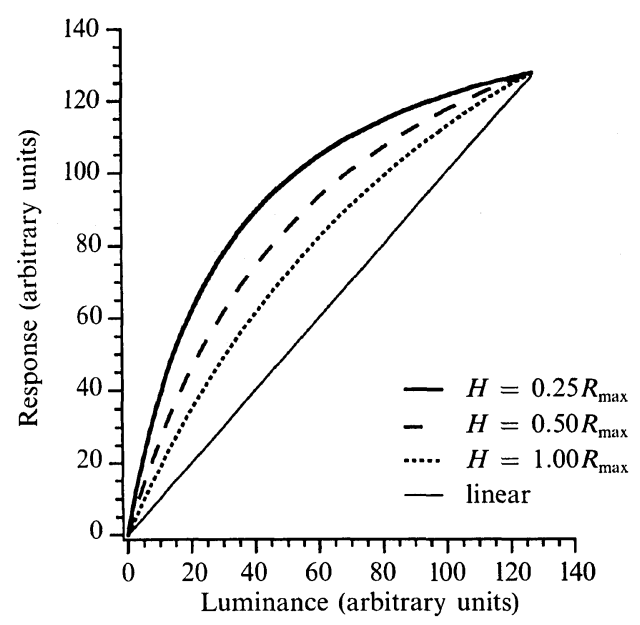

Figure 10. The $\mathrm{H}$ transform. The $x$-axis shows the image luminance and the $y$-axis the output of the $\mathrm{H}$ transform. The transform responses have been normalised to the (original) $0-128$ range.

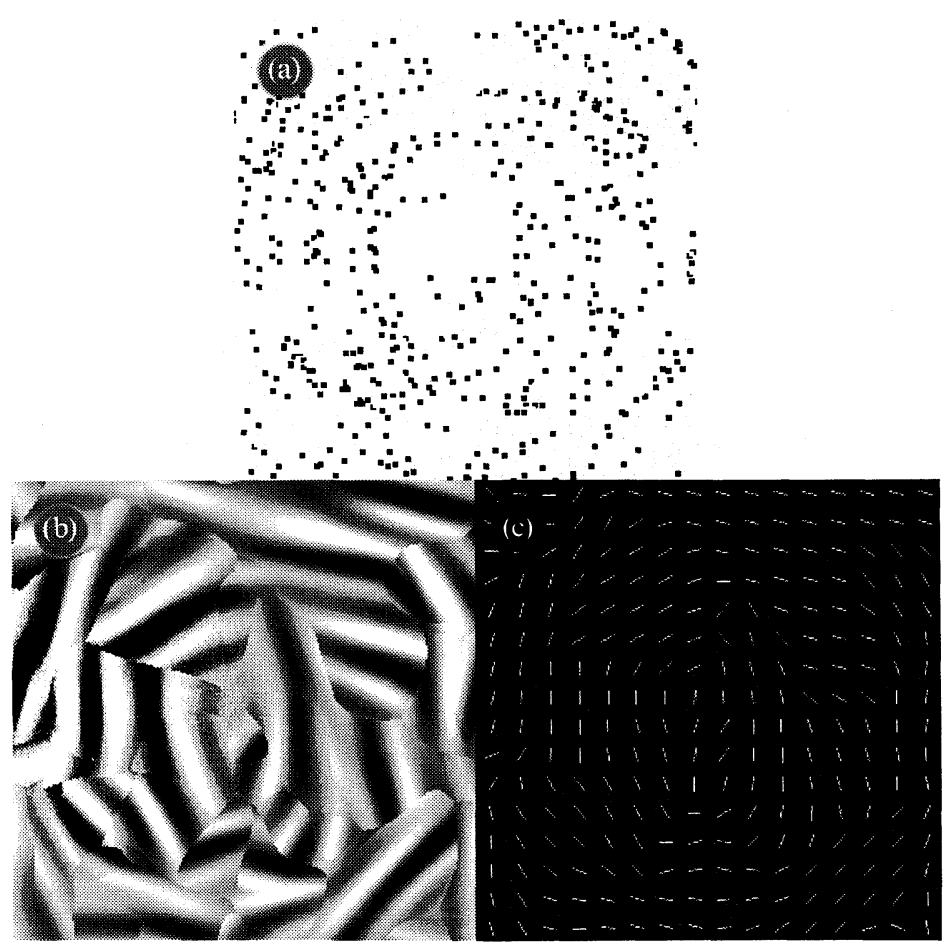

Figure 11. The effect of the $\mathrm{H}$ transform on the processing of interleaved patterns by the adaptive filtering model. (a) The H-transformed version of figure $9 \mathrm{a}$ for $H=0.5 R_{\max }$. The operation of the model on the output of the transformation (b), shown in the right half of the figure, produces the correct flow field (c). 
The operation of the $\mathrm{H}$ transform is shown in figure 10 which illustrates its effect of compressing high image intensities into a smaller band of responses leaving a larger range for the lower intensities. The result of applying such a nonlinearity to the interleaved patterns described above is to shift the luminance of the background towards that of the bright-feature set. Thus the bright features will interfere less with the filters' grouping of the dark set. Figure 11 shows the result of running the adaptive filtering procedure on an interleaved pattern which has been $\mathbf{H}$ transformed with values of $H=0.50$ (the maximum value of $H$ producing a veridical flow field). It is clear that, for this pattern at least, values of $H$ around 0.50 produce correctly oriented structure from the model. This implies that a rather severe nonlinearity is preceding filtering.

Perceived flow switches abruptly as the severity of the nonlinearity is changed; there is no intermediate flow between a rotational and a dilational field. According to Prazdny (1986), bistability is a necessary feature of a model of these phenomena and is one that purely linear filtering models do not have. This demonstration suggests that the bistability of these patterns, reported by some observers, is the result of change in this early nonlinearity This, in turn, could be due to something as basic as retinal adaptation, depending on where one locates this nonlinearity in the visual system. Comparing figures 9 and 11 shows that it is very-coarse-scale filters which are used to calculate the correct orientation structure of these patterns. This allows the clear prediction that perception of structure should be significantly impaired, compared to perception of structure in single-contrast patterns, in the presence of a spatially lowpass mask, since intermediate scales are of no use for deriving orientation structure.

\subsection{A 'second-order' mechanism?}

Recently there has been increasing interest in our ability to see structure in textures which cannot be detected by (first-order) linear filtering mechanisms ('second-order' texture perception; Sutter et al 1995). Sutter et al used a cârrier consisting of spatially band-pass random noise which was contrast-modulated by a Gabor envelope. Their proposed mechanism for detecting structure in these patterns are second-order filters, operating on the rectified output of a linear filter. Sutter et al (1995) showed that such mechanisms appear to be tuned to frequencies roughly $8-16$ times lower than the carrier.

Consider the elements comprising an interleaved Glass pattern, such as figure $8 \mathrm{a}$, as being approximated by two cycles of a vertical sinusoid modulated by a highly elongated horizontal contrast envelope. This is illustrated in figure 12a, which shows a pattern composed of sinusoidal carrier patches, oriented orthogonal to a rotational flow field, and each modulated by a Gaussian contrast envelope oriented parallel to the flow field. The impression of orientation structure is dominated by the envelope orientation. What appears to be happening is that the visual system is getting two sources of local orientation information: first-order information about the carrier, and second-order information about the envelope. In order to make sense of these two sources of orientation information it is likely that those estimates are accompanied by an estimate of their reliability (which equates to orientational bandwidth). For the carrier in figure $12 \mathrm{a}$ reliability is low (ie bandwidth is high), but for the envelope reliability is high. Thus the visual system weights the contribution of the envelope more heavily and it dominates the percept of flow. How the envelope of these elements is extracted is debatable, although popular mechanisms are to half-wave or full-wave rectify filter outputs and then feed this into another bank of filters.

If the visual system is weighing up the contribution of first-order and second-order information to estimate local orientation, it might be possible to see interactions between the two as their relative bandwidths change. The simplest way to do this, for the patterns shown, is to rotate the carrier relative to the envelope orientation which will steadily increase the relative reliability of the carrier, as well as its consistency 


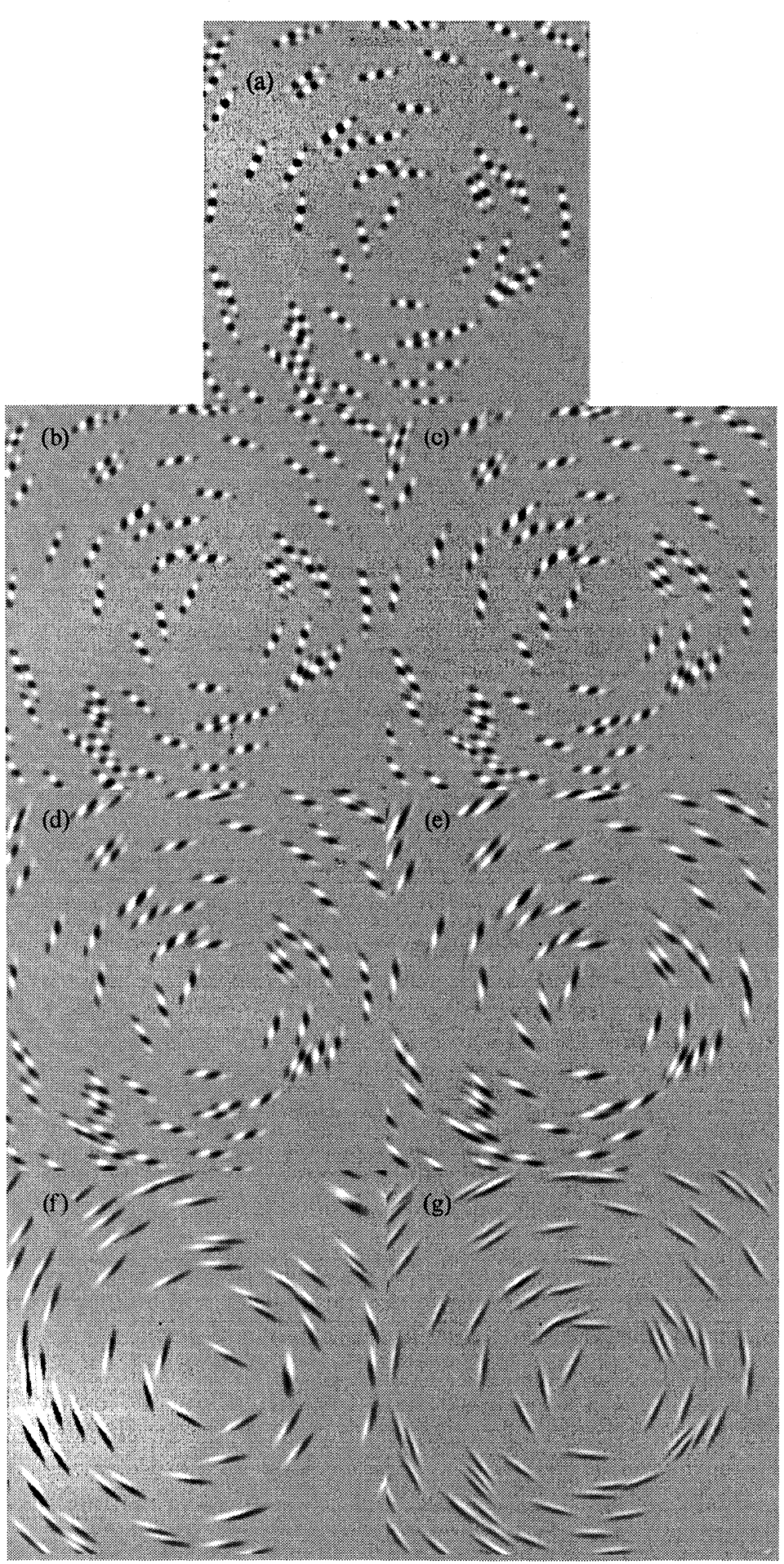

Figure 12. (a) Texture composed of envelopes, oriented according to a rotational flow field, modulating sinusoidal carriers oriented orthogonal to the local orientation of the flow field. (b) - (g) Effect of rotating the carrier towards the envelope orientation. 
with the envelope. The remainder of figure 12 shows the effect of doing this. As the carrier rotates, the two sources of information are combined, skewing local orientation estimates and producing a percept of a spiral (figures 12c, 12d, and 12e). As the two come into registration (figures $12 \mathrm{f}$ and $12 \mathrm{~g}$ ) the correct rotational structure is again perceived. This demonstration suggests that local orientation estimates may be formed by the weighted vector sum of first-order and second-order estimates.

This demonstration is clearly closely related to the Fraser 'twisted cord' illusion (Fraser 1908; Morgan and Moulden 1986). Indeed, a proposed explanation for the Fraser effect is in terms of 'collector units' which receive inputs from similarly oriented receptive fields lying along a path roughly consistent with their preferred orientation/ position (Morgan and Moulden 1986). This is effectively a second-order orientation mechanism. The degree of similarity between the orientation of subunits and the orientation of the path over which they are integrated effectively determines a weighting function for the contribution of the first-order input to the estimated orientation.

\section{Discussion}

The purpose of this paper has been to show that criticisms of spatial-filtering models of grouping using contrast phenomena tend to be based on one of two tenuous suppositions: that all visual processing preceding filtering is entirely linear, or that the grouping is achieved by means of low-pass filtering. It has been shown that spatially band-pass mechanisms are capable of deriving structure from 'balanced' Glass patterns. The adaptive filtering model correctly predicts that, for dense patterns composed of opposite contrast pairs, local flow direction will be rotated by $90^{\circ}$. Psychophysical data from a study by Kovacs and Julesz (1992), quantifying this effect, have also been shown to be consistent with this model.

The most problematic, and consequently the most interesting, phenomenon associated with Glass patterns remains the veridical perception of structure in interleaved opposite-contrast patterns. I have described two mechanisms for deriving structure from these patterns: through the addition to the adaptive filtering model of an early (albeit fairly severe) nonlinearity, and through the addition of a second stage of filtering operating on the rectified output of first-order mechanisms.

With respect to nonlinear contrast transduction, a comprehensive explanation of how such a nonlinearity might be incorporated into the model has not been provided, since it has been suggested that the severity of this function may be affected by retinal adaptation (Morgan et al 1984) and by local mean luminance (Watt 1991). The problems of dealing with these factors go beyond the scope of this paper. The second possibility, that the visual system has continuous access to first-order and second-order estimates of orientation, leads to the question of how those estimates might be combined. The demonstration illustrated in figure 12 suggests that some form of vector summation, weighted by estimates of local orientational bandwidth, might suffice. Given that little is at present known about how first-order and second-order information is combined, the use of textures composed of independently oriented carriers and envelopes may be a useful way of examining this problem.

Acknowledgements. I thank Roger Watt and Ian Paterson for many helpful discussions of this work, and Robert Hess for his comments on a draft of this paper. Thanks also to Dave Earle who introduced me to Glass patterns. This work was supported by SERC (grant GR/H53181) while the author was at the University of Stirling.

\section{References}

Anstis S, 1970 "Phi movement as a subtraction process" Vision Research $101411-1430$

Beck J, Sutter A, Ivry R, 1987 "Spatial frequency channels and perceptual grouping texture segmentation" Computer Vision Graphics and Image Processing 37 299-325

Caelli T, Julesz B, 1979 "Psychophysical evidence for global feature processing in visual texture discrimination" Journal of the Optical Society of America A 69 675-678 
Campbell F, Robson J 1968 "Application of Fourier analysis to the visibility of gratings" Journal of Physiology (London) $197551-566$

Carlson C, Anderson C, Moeller J, 1980 "Visual illusions without low spatial frequencies" Investigative Ophthalmology and Visual Science, Supplement 19165

Dakin S C, in press "The detection of structure in Glass patterns: Psychophysics and computational models" Vision Research

Fraser J, 1908 "A new illusion of visual direction" British Journal of Psychology 2 307-320

Garcia-Perez M A, 1991 "Visual phenomena without low spatial frequencies: a closer look" Vision Research $311647-1653$

Gelb D, Wilson H, 1983 "Shifts in perceived size as a function of contrast-and temporal modulation" Vision Research 23 71-82

Glass L, 1969 "Moiré effects from random dots" Nature (London) 243 578-580

Glass L, Switkes E, 1976 "Pattern recognition in humans: Correlations which cannot be perceived" Perception 5 67-72

Graham N, Beck J, Sutter A, 1992 "Nonlinear processes in spatial-frequency channel models of perceived texture segregation: Effects of sign and amount of contrast" Vision Research 32 $719-743$

Hubel D, Wiesel T, 1967 "Receptive fields, binocular interaction and functional architecture in the cats' visual cortex" Journal of Physiology (London) $160106-154$

Janez L, 1984 "Visual grouping without low spatial frequencies" Vision Research 24 271-274

Kass M, Witkin A, 1985 "Analyzing oriented patterns", in Proceedings of the Ninth International Joint Conference on Artificial Intelligence (Los Angeles, CA) pp 944-952 [reprinted in Readings in Computer Vision: Issues, Problems, Principles, and Paradigms Eds M A Fischler, O Fischlein (1987, Los Altos, CA: Morgan Kaufmann) pp 268-276]

Kovacs I, Julesz B, 1992 "Depth motion and static-flow perception at metaisoluminant color contrast" Proceedings of the National Academy of Sciences of the USA $8910390-10394$

Legge G, Kersten D, 1983 "Light and dark bars: Contrast discrimination" Vision Research 23 473 - 483

Marr D, 1976 "Early processing of visual information" Proceedings of the Royal Society of London B $275483-534$

Morgan M J, Mather G, Moulden B, Watt R J, 1984 "Intensity-response nonlinearities and the theory of edge localization" Vision Research 24 713-719

Morgan M J, Moulden B, 1986 "The Münsterberg figure and twisted cords" Vision Research 26 $1793-1800$

Naka K, Rushton W, 1966 "S-potentials from luminosity units in the retina of fish 'cyprinidae" Journal of Physiology (London) 185 587-599

Palmer S, 1992 "Modern theories of Gestalt perception", in Understanding Vision Ed. G Humphreys (Oxford: Basil Blackwell) pp 39-70

Prazdny K, 1986 "Some new phenomena in the perception of Glass patterns" Biological Cybernetics $53153-158$

Reed T, Wechsler H, 1990 "Segmentation of textured images and Gestalt organisation using spatial/ spatial-frequency representations" IEEE Transactions on Pattern Analysis and Machine Intelligence $121-12$

Shapley R, Victor J, 1979 "The contrast gain control of the cat retina" Vision Research 19 431-434

Stevens K, 1978 "Computation of locally parallel structure" Biological Cybernetics 6 19-28

Stevens K, Brookes A, 1978 "Detecting structure by symbolic constructions on tokens" Computer Vision Graphics and Image Processing $371133-1145$

Sutter A, Sperling G, Chubb C, 1995 "Measuring the spatial frequency selectivity of secondorder texture mechanisms" Vision Research 35 915-924

Tolhurst D, Thompson P, 1975 "Orientation illusions and aftereffects: Inhibition between channels" Vision Research $5967-972$

Watt R J, 1988 Visual Processing: Computational Psychophysical and Cognitive Research (London: Lawrence Erlbaum Associates)

Watt R J, 1991 Understanding Vision (London: Academic Press)

Zucker S, 1982 Early Orientation Selection and Grouping: Evidence for Type I and Type II Processes (Montreal: McGill University)

Zucker S W, Davis S, 1988 "Points and endpoints: A size/spacing constraint for dot grouping" Perception 17 229-247

Zucker S W, Stevens K A, Sander P, 1983 "The relation between proximity and brightness similarity in dot patterns" Perception \& Psychophysics 34 513-522 